\title{
Surveys of catheter-associated urinary tract infection in a university hospital intensive care unit in China
}

Authors

Duo-shuang Xie ${ }^{1}$

Rui-ping $\mathrm{Lai}^{2}$

Shao-fa $\mathrm{Nie}^{3}$

${ }^{1} \mathrm{PhD}$; Department of Epidemiology and Biostatistics, School of Public Health, Tongji Medical College, Huazhong University of Science and Technology, Wuhan, China; Department of Infection Control, Taihe Hospital, Hubei University of Medicine, Hubei, China ${ }^{2} \mathrm{MD}$; Department of Infection Control, Taihe Hospital, Hubei University of Medicine, Hubei, China ${ }^{3} \mathrm{MSc}$; Department of Epidemiology and Biostatistics, School of Public Health, Tongji Medical College, Huazhong University of Science and Technology, Wuhan, China

Submitted on: 12/31/2010 Approved on: 01/13/2011

\footnotetext{
Correspondence to:

Shao-fa Nie

Department of

Epidemiology and

Biostatistics

School of Public Health,

Tongji Medical College

Huazhong University of

Science and Technology

13 Hangkong Road,

Wuhan, Hubei 430030,

P.R.China.

tjnsf2007@163.com
}

We declare no conflict of interest.
In developed nations, urinary tract is the most common infection site among healthcare-associated infection (HCAI). ${ }^{1}$ In a report of point prevalence surveys of HCAI conducted in 13 hospitals in China, urinary tract infection (UTI) was the third most common infection. ${ }^{2}$ Catheter-associated urinary tract infection (CAUTI) has been common nosocomial infection around the world. Most published studies of CAUTI originated from hospitals in developed nations. Relatively few data is available from China to the present CAUTI epidemic situation. The present study was designed to determine the prevalence and explore the important risk factors of CAUTI in an ICU in a tertiary care university hospital.

A cohort of 424 ICU patients with indwelling urethral catheters was observed between October 2007 and June 2008. Of them 285 were male and 139 were female. Their mean age was 48.3 years ranging from 15 to 84 years. During the patients stay in the ICU, sterile technique was applied to collect urine for bacterial culture and further colony count every other day from the first day of indwelling catheter. Medical records were reviewed and registered by the ICU senior physicians for CAUTI, using the $\mathrm{CDC}$ case definitions. Asymptomatic bacteriuria was not computed as UTI in the present report.

These 424 patients contributed with 2,632 catheter-days and 3,981 patient-days. The number of catheters in use was 71.9 per 100 patients (or 661 catheter-days per 1000 patient-days). The incidence rate of CAUTI was $15.8 \%$ (67/424, 95\% CI: $12.33 \%-19.27 \%)$, and the density of infection was $25.46(67 / 2632$, 95\% CI: 19.44-31.47) per 1,000 catheter-days. The incidence density was higher than those in ICUs from both developed and developing countries. ${ }^{1,3}$ By the multinomial logistic regression analysis, CAUTI risk factors were duration of catheterization ( $\geq 7$ days), benign prostatic hypertrophy, and duration of antimicrobial treatment $\geq 5$ days before the onset of CAUTI, with ORs of $10.23(95 \%$ CI: 5.18 20.24), 7.23(95\% CI: $1.58-33.17$ ), and $4.17(95 \%$ CI: 1.79-9.72), respectively. Increased duration of catheterization was associated with UTI incidence in the ICU. When the duration of catheterization was longer than 10 days, UTI occurred in almost 100\% ICU patients. Antimicrobial treatment might suppress the endogenous bacterial flora of the urinary system, and then lead to CAUTI. ${ }^{4}$ Benign prostatic hypertrophy was an age-related disease and could cause anatomical and physiological change and urine outflow obstruction. In this cohort, $65.67 \%$ (44/67) of CAUTI yielded positive results by bacteriologic tests. There were 47 pathogenic bacteria isolated. Of these isolates, fungi were the most frequently isolated (21.28\%, 10/47), followed by Escherichia coli (17.02\%, 8/47), and Pseudomonas aeruginosa (10.64\%, 5/47). Escherichia coli resistance for ciprofloxacin was $88 \%$, and Pseudomonas aeruginosa showed absolute resistance to ciprofloxacin, amikacin, ceftazidime and meropenem.

Once the patients started to be evaluated daily by using appropriate criteria for catheter continuation and timely removal, the duration of catheterization was significantly reduced and the incidence of CAUTI decreased significantly. ${ }^{5}$ Full-effective measures of infection control should be currently designed and implemented to reduce the prevalence of CAUTI in the ICU. [Braz J Infect Dis 2011;15(3):296-297] @Elsevier Editora Ltda.

\section{REFERENCES}

1. Edwards JR, MStat, Peterson KD et al. National Healthcare Safety Network (NHSN) Report, data summary for 2006 through 2007, issued November 2008. Am J Infect Control. 2008; 36:609-26.

2. Xie DS, Xiong W, Xiang LL et al. Point prevalence surveys of healthcare-associated infection in 13 hospitals in Hubei Province, China, 20072008. J Hosp Infect. 2010; 76:150-5. 
3. Talaat M, Hafez S, Saied T, Elfeky R, El-shoubary W, Pimentel G. Surveillance of catheter-associated urinary tract infection in 4 intensive care units at Alexandria university hospitals in Egypt. Am J Infect Control. 2010; 38:222-8.

4. Alvarez-Lerma F, Nolla-Salas J, Leon C et al. Candiduria in critically ill patients admitted to intensive care medical units. Intens Care Med. 2003; 29:1069-76.
5. Elpern EH, Killeen K, Ketchem A, Wiley A, Patel G, Lateef O. Reducing use of indwelling urinary catheters and associated urinary tract infections. Am J Crit Care 2009; 18:535-41. 